\title{
Collagenofibrotic Glomerulopathy: Report a Rare Case
}

\author{
Rakesh Agarwal, MD'; Rashmi Baid, MS²; Jotideb Mukhopadhyay, MD \\ 'Institute of Post-Graduate Medical Education and Research and Seth Sukhlal Karnani Memorial Hospital, Kolkata, India \\ ${ }^{2}$ Lilavati Hospital \& Research Centre, Mumbai, India
}

\begin{abstract}
Collagenofibrotic glomerulopathy is a rare clinical entity with fewer than 40 cases reported worldwide. We describe a case of adult onset nephrotic syndrome with partial villous atrophy of the intestine who was diagnosed with this rare entity. Collagenofibrotic glomerulopathy is characterized by subendothelial and mesangial collagen type III deposition and increased procollagen III peptide levels. Extra renal involvement has been described in the form of hypertension, anemia and microangiopathic hemolytic anemia but villous atrophy has not been associated with this condition so far, possibly reflecting the paucity of literature. We describe this case and review the condition in this report.

Keywords: Collaagenofibrotic glomerulopathy, Nephrotic syndrome

Cite this article as: Agarwal R, Baid R, Mukhopadhyay J. Collagenofibrotic glomerulopathy: report a rare case. Arch Iran Med. 2020;23(6):409-411. doi: 10.34172/aim.2020.35.
\end{abstract}

Received: July 29, 2018, Accepted: February 11, 2019, ePublished: June 1, 2020

\section{Introduction}

Collagenofibrotic glomerulopathy, first identified by Arakawa et al is a rare clinico-pathological entity with fewer than 40 cases reported worldwide. ${ }^{1}$ It is characterized by subendothelial and mesangial collagen type III deposition and increased procollagen III peptide levels. It presents with proteinuria, hematuria and hypertension and can lead to renal failure of varying degrees. ${ }^{2}$ It is considered to be a newly described hereditary glomerulopathy and not just a type of nail-patella syndrome as previously thought. ${ }^{3}$ We describe a rare case of recent-onset nephrotic syndrome which was diagnosed as collagenofibrotic glomerulopathy on renal biopsy and had partial villous atrophy on gastrointestinal endoscopy and guided biopsy. This is the first case report of such an association, to the best of the authors' knowledge.

\section{Case Report}

A 32-year old male patient, recently detected to be hypertensive, presented to the medicine outpatient department with progressively increasing edema and facial puffiness over the last several weeks. He had no similar history in the past. There was not any significant family history related to the condition.

His blood pressure on admission was 152/74 mm Hg and he was mildly anemic. No bone or nail dysplasia was noted. He had no visual or hearing abnormalities.

Urinalysis revealed marked proteinuria $(12 \mathrm{~g} / \mathrm{d})$, with no pathological sediments. Bence Jones protein was absent in urine. His hemoglobin level was $11.8 \mathrm{~g} / \mathrm{dL}$, and WBC count was 7400 cells $/ \mathrm{mm}^{3}$ with normal differentiation.
Serum total protein was $2.8 \mathrm{~g} / \mathrm{dL}$, with $1.6 \mathrm{~g} / \mathrm{dL}$ albumin and $1.2 \mathrm{~g} / \mathrm{dL}$ globulin. His serum creatinine was 1.3 $\mathrm{mg} / \mathrm{dL}$, and serum urea was $37 \mathrm{mg} / \mathrm{dL}$. Liver function tests, blood glucose, serum electrolytes, and HbA1C were normal. Serum protein electrophoresis showed no monoclonal peak. Thyroid function tests were negative.

His low albumin and globulin levels prompted us to undertake an upper gastrointestinal endoscopy with guided duodenal biopsy. This revealed partial villous atrophy. However, no specific pattern could be identified. Tissue trans-glutaminase and serum antinuclear antibody tests were negative.

An ultrasound guided renal biopsy was carried out. It revealed accumulation of large amounts of acellular material in the mesangium, causing enlargement of the glomeruli. This material was weakly PAS positive, silver negative and blue on trichrome stain (Figure 1). Congo Red staining for amyloid was negative. No significant interstitial fibrosis and tubular atrophy was seen. Mild fibrous intimal proliferation of arteries was noted. Immunofluorescence stains for IgG, IgM, IgA, C3, C1q, kappa and lambda light chains were negative. These findings were suggestive of collagenofibrotic glomerulopathy.

The patient was put on oral prednisolone therapy ( 40 $\mathrm{mg} / \mathrm{d}$ ) and diuretics. However, he chose not to undergo treatment after three months and was lost to follow-up.

\section{Discussion}

First reported in 1979 as "idiopathic mesangio-degenerative glomerulopathy", collagenofibrotic glomerulopathy (CG) is an extremely rare glomerular disease. ${ }^{1}$ Fewer than 20 


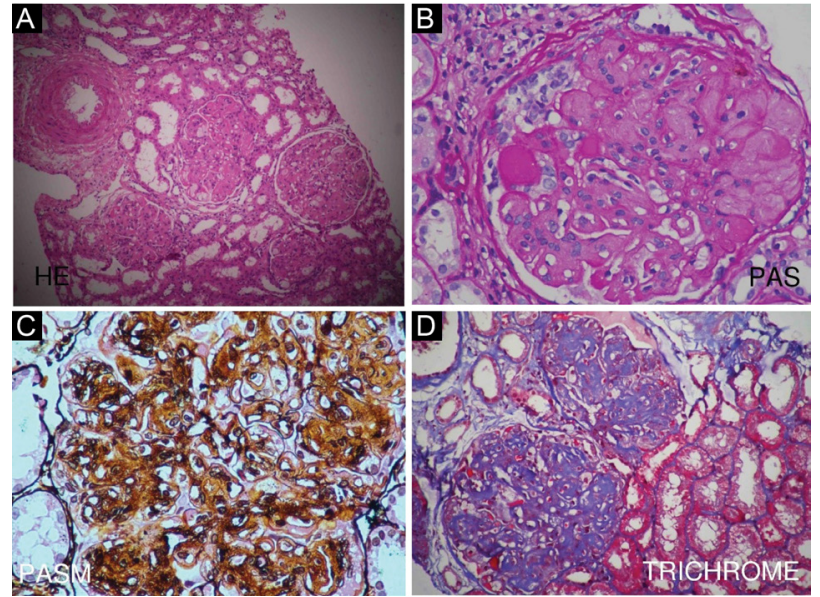

Figure 1. (A) HE Stain Showing Collagenofibrotic Glomerulopathy. (B) PAS Positive Staining of the Biopsy. (C) PASM Staining of the Biopsy. (D) Blue Staining on Mason Trichrome.

cases have been described from India. ${ }^{4}$ Being a rare entity, the exact pathogenesis and etiology of the disease remain unknown.

It is usually sporadic, though inheritance as an autosomal recessive trait has been suggested. Out of the 38 reported cases, 16 patients have been of Japanese descent. This suggests that environmental or ethnic factors may play a role in causation of the disease. Familial cases have been described which, on the contrary, points to a genetic etiology.

In addition, it remains unknown if $\mathrm{CG}$ is a primary kidney disease or occurs secondary to a systemic disorder. The mRNA in renal mesangial cells codes for collagen type III. Alterations cause them to produce more collagen III. ${ }^{6,7}$ Extra-renal involvement has been described in CG as in our case, which also suggests a systemic nature for the disease. ${ }^{7,8}$

The clinical picture of CG is varied. All age groups and both sexes are affected. The highest incidence is noted in the $4^{\text {th }}$ to $7^{\text {th }}$ decades of life, but children below 15 years are also known to have been affected. Most pediatric cases have been reported from France. ${ }^{3,7}$ CG presents commonly with persistent proteinuria and edema. More than half have nephrotic range proteinuria. Hypertension is early and is seen in two-thirds of cases at presentation. ${ }^{9-11}$ Anemia and microangiopathic hemolytic anemia have been described. ${ }^{12}$ Cases have been described in association with factor $\mathrm{H}$ deficiency, and extra renal involvement. ${ }^{13}$ Symptoms progress steadily to eventual renal failure.

Laboratory work-up yields nonspecific results with normal or slightly deranged kidney function. Urinalysis may reveal proteinuria and microscopic hematuria but lacks active sediment. PIII P levels are elevated and reach up to 100 times that normally seen in healthy people. This implicates excessive production of, or altered type II collagen metabolism in the pathology of the disease. ${ }^{12,14}$

Light microscopy shows globally enlarged glomerular tufts due to the eosinophilic material in capillary walls and mesangium. It is weakly PAS positive. Periodic acidmethenamine silver, aniline blue, and mason trichrome staining show strong blue staining. Congo red and thioflavin $\mathrm{T}$ staining are negative. Advanced cases show narrowed capillary lumina due to mesangial expansion and capillary wall thickening. Immunofluorescence staining is usually negative. ${ }^{7,12,14}$

Immunohistochemistry shows abundant type II collagen staining. Electron microscopy shows greatly increased fibrillary material in subendothelium of basement membrane and mesangium. In nail-patella syndrome, banded collagen profiles deposit mainly in the lamina densa. This differential collagen deposition distinguishes the two entities. The banded collagen morphology in CG can be identified better with special stains tannic acid lead and phosphotungstic acid. The pattern tends to be irregular bundles with curved or frayed fibers rather than straight fibers. ${ }^{7,12,14}$

No specific therapy for CG is available so far. Supportive management with control of hypertension and edema is needed. Patients who develop end-stage renal failure need dialysis or renal transplantation. Data on recurrence in the transplanted kidney is limited. The role of systemic glucocorticoids is unclear, and considering the rarity of the disease, a future trial is not in foresight. Prednisolone may be used to treat proteinuria, renal dysfunction and anemia and is said to decrease serum PIII P levels. Dipyridamole therapy has also been used..$^{15,16}$

Our case is unique in that it not only adds to the scarce literature on the subject but is the first case that documents the intestinal association of the disease. The outcome of the patient could not be followed up. However, it does educate us to keep a close eye on other systems when dealing with such a rare disease entity in the future.

\section{Authors' Contribution}

$\mathrm{RB}$ and RA participated in data collection, data analysis, review of literature, structure of the article, writing of the manuscript, and direct patient care. JM participated in patient management, editing of the manuscript, structure of the article, and final evaluation of manuscript.

\section{Conflict of Interest Disclosures}

The authors report no conflicts of interest.

\section{Ethical Statement}

Informed consent was taken form the patient for publication of the report.

\section{References}

1. Arakawa M, Hueki H, Sato M, Yamashita K, Nakashima S. Idiopathic mesangiodegenerative glomerulonephropathy: A proposal of a new glomerular disease. Jpn J Nephrol. 1979;21:1404.

2. Gibson IW, More IA. Glomerular pathology: recent advances. J Pathol. 1998;184(2):123-9. doi: 10.1002/(SICl)10969896(199802)184:2<123::AID-PATH16>3.0.CO;2-X.

3. Gubler MC, Dommergues JP, Foulard M, Bensman A, Leroy JP, Broyer M, Habib R. Collagen type III glomerulopathy: 
a new type of hereditary nephropathy. Pediatr Nephrol. 1993;7(4):354-60. doi: 10.1007/BF00857536.

4. Nimmagadda S, Mukku K, Devaraju SR, Uppin MS. Unusual cause of glomerular deposition disease: collagenofibrotic glomerulopathy. Indian J Nephrol. 2017;27(1):62-5. doi: 10.4103/0971-4065.179300.

5. Tamura H, Matsuda A, Kidoguchi N, Matsumura O, Mitarai $\mathrm{T}$, Isoda K. A family with two sisters with collagenofibrotic glomerulonephropathy. Am J Kidney Dis. 1996;27(4):588-95. doi: 10.1016/s0272-6386(96)90172-9.

6. Scheinman JI, Tanaka H, Haralson M, Wang SL, Brown O. Specialized collagen mRNA and secreted collagens in human glomerular epithelial, mesangial, and tubular cells. J Am Soc Nephrol. 1992 Apr;2(10):1475-83.

7. Duggal R, Nada R, Rayat CS, Rane SU, Sakhuja V, Joshi K. Collagenofibrotic glomerulopathy- a review. Clin Kidney J. 2012;5(1):7-12. doi: 10.1093/ndtplus/sfr144.

8. Mizuiri S, Hasegawa A, Kikuchi A, Amagasaki Y, Nakamura $\mathrm{N}$, Sakaguchi H. A case of collagenof ibrotic glomerulopathy associated with hepatic perisinusoidal fibrosis. Nephron. 1993;63(2):183-7. doi: 10.1159/000187180.

9. Imbasciati E, Gherardi G, Morozumi K, Gudat F, Epper R, Basler V, et al. Collagen type III glomerulopathy: a new idiopathic glomerular disease. Am J Nephrol. 1991;11(5):4229. doi: 10.1159/000168350.
10. Bernasovská G, Demes M, Oksa A, Pavlovic M, Vahancík A, Nyitrayová $\mathrm{O}$, et al. Collagenofibrotic glomerulopathy--rare glomerulonephritis. Vnitr Lek. 2006;52(12):1200-4. [Czech].

11. Ferreira RD, Custódio FB, Guimarães CS, Corrêa RR, Reis MA. Collagenofibrotic glomerulopathy: three case reports in Brazil. Diagn Pathol. 2009;4(1):33. doi :10.1186/1746-1596-4-33.

12. Patro KC, Jha R, Sahay M, Swarnalatha G. Collagenofibrotic glomerulopathy- case report with review of literature. Indian J Nephrol. 2011;21(1):52-55. doi: 10.4103/0971-4065.78080.

13. Vogt BA, Wyatt RJ, Burke BA, Simonton SC, Kashtan CE. Inherited factor $\mathrm{H}$ deficiency and collagen type III glomerulopathy. Pediatr Nephrol. 1995;9(1):11-5. doi: 10.1007/BF00858956.

14. Alchi B, Nishi S, Narita I, Gejyo F. Collagenofibrotic glomerulopathy: clinicopathologic overview of a rare glomerular disease. Am J Kidney Dis. 2007;49:499-506. doi: 10.1053/j.ajkd.2007.01.020.

15. Hisakawa N, Yasuoka N, Nishiya K, Kumon Y, Okamoto $\mathrm{K}$, Itoh $\mathrm{H}$, et al. Collagenofibrotic glomerulonephropathy associated with immune complex deposits. Am J Nephrol. 1998;18(2):134-41. doi: 10.1159/000013322.

16. Yoshida F, Yuzawa Y, Shigematsu H, Ito A, Yamazaki C, Yoshioka $\mathrm{K}$, et al. Nephrotic syndrome with massive accumulation of type I and type III collagen in the glomeruli. Intern Med. doi: 1993;32:171-6. doi: 10.2169/internalmedicine.32.171. 